Annuaire suisse de politique de développement

16 | 1997

Environnement et développement, 5 ans après Rio

\title{
Coopération et transfert de technologie dans le domaine de l'environnement
}

Andràs November

\section{(2) OpenEdition}

1 Journals

Édition électronique

URL : http://journals.openedition.org/aspd/815

DOI : 10.4000/aspd.815

ISSN : 1663-9669

Éditeur

Institut de hautes études internationales et du développement

Édition imprimée

Date de publication : 1 mars 1997

Pagination : 247-254

ISSN : 1660-5934

Référence électronique

Andràs November, "Coopération et transfert de technologie dans le domaine de l'environnement »,

Annuaire suisse de politique de développement [En ligne], 16 | 1997, mis en ligne le 08 août 2012,

consulté le 08 septembre 2020. URL : http://journals.openedition.org/aspd/815 ; DOI : https://doi.org/ $10.4000 / a s p d .815$

(c) The Graduate Institute I Geneva 


\title{
COOPÉRATION ET TRANSFERT DE TECHNOLOGIE DANS LE DOMAINE DE L'ENVIRONNEMENT
}

\author{
ANDRÀS NOVEMBER
}

\begin{abstract}
a Conférence des Nations Unies sur l'environnement et le développement, qui s'est tenue à Rio en 1992, a mis en exergue l'importance des « écotechnologies " dans le processus de développement durable (sustainable development), de même que la nécessité de faciliter leur transfert vers les pays du tiers monde.'
\end{abstract}

La prise de conscience de la dégradation de l'environnement, dont les principaux symptômes sont le réchauffement de l'atmosphère et les changements climatiques, la destruction de la couche d'ozone, l'effet de serre, la déforestation et la diminution de la biodiversité, constitue le fondement des technologies environnementales. Leur diffusion comporte un aspect solidaire, étant donné que leur utilisation dépasse l'intérêt économique de l'acheteur ou du vendeur de la technologie.

\section{DES TECHNOLOGIES APPROPRIÉES AUX TECHNOLOGIES ENVIRONNEMENTALES}

L'idée de technologies compatibles avec l'environnement naturel s'inscrit dans la mouvance des critiques formulées à l'encontre des technologies industrielles lourdes, polluantes, comportant des risques élevés. Les adeptes des «technologies appropriées » préconisent, dès les années soixante, l'utilisation optimale des matières premières, le recours à des procédés de production non polluants et aux énergies « douces ». Elles favorisent la diversification, la décentralisation et les petites unités industrielles intensives en main-d'œuvre, afin de créer des conditions économiques, écologiques et sociales optimales. Le contrôle social et l'évaluation des risques technologiques vont de pair avec les choix technologiques. Les technologies de l'environnement sont la concrétisation des technologies appropriées pour promouvoir le développement durable - sustainable development - qui postule la compatibilité économique, écologique et sociale des décisions politiques. Le transfert de technologies environnementales devient ainsi un des vecteurs de l'intégration de la protection de l'environnement dans les stratégies de développement. Définies dans le chapitre 34 de l'Agenda 21 de la Conférence de Rio, ces technologies sont «moins polluantes, utilisent de façon plus durable les ressources, autorisent le recyclage de leurs déchets et produits, ainsi que le traitement des déchets résiduels d'une manière plus acceptable que les technologies qu'elles remplacent $»^{2}$.

1 Il existe de nombreuses expressions pour désigner les «écotechnologies», notamment les « technologies respectueuses de l'environnement», les «technologies écologiquement rationnelles», les technologies «vertes», " propres ", "saines », etc. Nous utiliserons dans cette contribution l'expression de «technologies de l'environnement », comme terme générique.

2 «Transfer of environmentally sound technology, cooperation and capacity-building », Agenda 21 - United NationsProgramme of Action, United Nations, New York, 1992, p. 252. 
Les industries de l'environnement sont en plein essor. ${ }^{3}$ Selon une estimation de l'OCDE, le marché mondial des biens et services environnementaux dépasse 200 milliards de dollars par an, et atteindra 300 milliards de dollars en l'an 2000. En outre, cette technologie progresse au taux annuel moyen de $5 \%$ à $6 \%$ et offre de nombreuses places de travail. Les pays de l'OCDE représentent $85 \%$ de ce marché, qui est tributaire avant tout de l'introduction de normes environnementales de plus en plus sévères. ${ }^{4}$

Le développement des technologies environnementales est stimulé par le changement de comportement des entreprises, avec les grandes entreprises chimiques en tête. Si les entreprises agissent avant tout sous la contrainte des règlements et des normes légales de la protection de l'environnement, il s'avère que la législation n'est pas l'unique facteur qui les incite à adopter une attitude positive pour réduire la pollution provenant de leurs activités. L'évolution des valeurs sociétales et les critiques de la société industrielle exprimées par les mouvements associatifs amènent certaines entreprises - certes pas toutes - à revoir leur politique environnementale en vue d'améliorer leur image.

Toutefois, les technologies environnementales n'apportent pas de solutions toutes faites aux problèmes globaux de l'environnement. Pour augmenter sensiblement l'efficacité écologique des technologies, il faudra repenser tous les processus productifs (par exemple, fermer les cycles de production, prévoir des économies d'énergie supplémentaires et l'utilisation à une plus large échelle des ressources renouvelables, etc.). Par ailleurs, l'introduction des technologies de l'environnement exige souvent des investissements considérables que les industriels ont quelque difficulté à consentir. Il existe pourtant de nombreuses solutions technologiques pour réduire les pollutions qui ne sont pas coûteuses. Par exemple, dans le nettoyage des circuits électroniques imprimés, les chlorofluorocarbones (CFC destructeurs de la couche d'ozone) peuvent être remplacés simplement par de l'eau.

\section{MANQUE DE MOTIVATION ENVIRONNEMENTALE DANS LE TIERS MONDE?}

Les choix technologiques ont des répercussions environnementales à la fois locales et globales. Mais force est de constater que dans la plupart des pays en voie d'industrialisation, les problèmes environnementaux sont relégués au second plan par les stratèges du développement économique. Même si la pollution a atteint des degrés élevés dans les métropoles des pays en développement et constitue une menace pour la santé de centaines de milliers d'habitants, même si l'érosion du sol met en danger la survie des paysans, les mesures de protection de l'environnement sont insuffisantes ou inexistantes dans un grand nombre de pays du tiers monde. Les gouvernements de ces pays estiment qu'ils ont d'autres priorités que la protection de l'environnement.

L'adoption de méthodes de fabrication plus respectueuses de l'environnement n'est pas nécessairement le résultat d'une prise de conscience dans les pays en

\footnotetext{
${ }^{3}$ Les technologies de l'environnement comprennent deux catégories : soit elles interviennent pour réduire les émissions polluantes qui ont été « produites » aux cours de la fabrication d'un objet (cleaning technologies), soit elles sont intégrées dans les procédés de fabrication pour les rendre moins polluants (cleaner technologies).

4 Stevens Candice, « L'industrie de l'environnement », dans L'Observateur de l'OCDE, no 177, août-septembre 1992, pp. 26-28.
} 
développement, mais elle est souvent induite ou imposée de l'extérieur. C'est le cas quand l'introduction de nouvelles normes environnementales dans les pays industrialisés touchent par ricochet les exportations des pays en développement. Par exemple, lorsque le gouvernement allemand a interdit l'utilisation de la substance PCP (penta-chlorophenol) dans le tannage du cuir, les producteurs de cuir de l'Inde ont perdu d'un seul coup leur plus important marché d'exportation. Pour le regagner, l'Inde a interdit l'usage du PCP dans toute fabrication du cuir. En changeant les standards nationaux, le gouvernement indien a imposé la reconversion de ses industries du cuir, mais en même temps, leurs produits devenaient euro-compatibles sur le plan environnemental. L'investissement a été rapidement amorti, puisque les produits « propres » sont devenus plus concurrentiels sur tous les marchés des pays industrialisés. ${ }^{5}$

La source de changement d'attitude à l'égard de l'environnement se trouve donc davantage dans les pays industrialisés que dans les pays en développement. Si la communauté internationale souhaite que les pays en développement adoptent les technologies de l'environnement, elle doit contribuer à leur transfert. Les pays du tiers monde refusent de payer deux fois le prix de leur industrialisation et de leur équipement industriel qui, dans la plupart des cas, ont été achetés dans les pays industrialisés (comme par exemple les industries qui utilisent les CFC). C'est la raison pour laquelle le transfert de technologies de l'environnement vers les pays en développement est devenu l'un des points prioritaires de la Commission des Nations Unies sur le développement durable (Commission for Sustainable Development), chargée du suivi des résolutions de la Conférence de Rio. Elle prévoit que les pays en développement puissent avoir accès aux technologies non polluantes et qu'ils puissent bénéficier de la coopération (aide financière) des pays industrialisés. Ces technologies devraient être mises à disposition à un "prix abordable » et sur une base « équitable » pour tous les pays. Même les Etats-Unis souscrivent à ces principes. La loi sur l'incitation à l'exportation (Export Enhancement Act) encourage " le recours aux programmes destinés au soutien des exportations de biens et de services qui remédient aux nuisances potentielles sur l'environnement ", et l'Agence de protection de l'environnement américaine (Environment Protection Agency) veut « accélérer la diffusion de technologies environnementales innovatrices sur place et à l'étranger $»^{6}$.

Il faut cependant être conscient que l'on ne peut pas susciter la motivation pour protéger l'environnent dans les pays en développement par décret. Une telle stratégie doit s'appuyer sur la conviction locale et être aidée par la coopération internationale sur la base d'un partenariat.

Le projet « Ecofrig » constitue un exemple intéressant de collaboration Nord-Sud (Suisse, Allemagne et Inde) en association avec plusieurs organisations non gouvernementales et institutions de recherche. Il s'agit de la fabrication d'un réfrigérateur répondant aux critères environnementaux suivants :

- il ne doit pas contenir de composants organiques halogènes (dont l'effet destructeur de la couche d'ozone est démontré),

- il doit être économe en énergie, être robuste, facile à réparer et à recycler,

- sa fabrication ne doit utiliser que des produits renouvelables.

\footnotetext{
5 Jha Veena \& Texeira Ana Paola, Are Environementally Sound Technologies the Emperor's New Clothes? UNCTAD, Discussion Papers, $n^{2}$ 89, Geneva, 1994, p. 14.

6 US Environmental Protection Agency, Technology Innovation Strategy, Washington, 1994.
} 
Grâce à de nombreuses innovations technologiques, la fabrication d'un tel réfrigérateur écologique a été réalisée en Inde. La technologie de remplacement du CFC à base d'hydrocarbures (substance utilisée aussi bien pour la réfrigération que pour l'isolation) représente une alternative intéressante par rapport aux autres substituts chimiques développés par Du Pont qui détient le monopole de ce marché (par exemple, HFC 134a). Les réfrigérateurs à base d'hydrocarbures sont moins polluants et leur entretien peut être effectué par le secteur informel des pays en développement.

\section{POLITIQUE SUISSE DE TRANSFERT ET DE COOPÉRATION TECHNOLOGIQUES DANS LE DOMAINE DE L'ENVIRONNEMENT}

Les politiques de développement industriel adoptées par les pays en développement impliquent le recours systématique au transfert de technologies qui ne se borne pas à la transplantation des techniques, mais entraîne aussi le transfert de tous les éléments constitutifs du système industriel (consommation énergétique élevée, utilisation intensive des matières premières, pollution, déchets, etc.). Parallèlement, le régime juridique de la propriété industrielle perpétue la dépendance des pays importateurs de technologies, étant donné que ces derniers doivent acquérir les technologies à des conditions commerciales imposées par les détenteurs de technologies?

Quand on examine la politique suisse en matière de transfert de technologies, la première chose qui frappe, ce n'est pas l'absence d'une politique clairement formulée, mais plutôt le fait que la Suisse a plusieurs politiques parallèles qui ne sont pas forcément convergentes. Il existe en effet différentes instances fédérales qui sont concernées directement ou indirectement par le transfert de technologies, mais il n'y a pas de stratégie cohérente en la matière. Ce constat s'explique aisément par le fait que le transfert de technologies revient essentiellement au secteur privé. Les autorités fédérales ne font qu'encadrer - pour promouvoir - les investissements et les ventes d'équipements des entreprises suisses à l'étranger. Pour ce faire, elles ont recours à une panoplie d'instruments comme la Garantie contre les risques à l'exportation (GRE), la Garantie contre les risques de l'investissement (GRI) et d'autres mesures de politique économique et commerciale au titre de la coopération internationale, en particulier l'octroi de crédits mixtes.

Le transfert de technologies environnementales suit le même schéma, tout en essayant d'adopter et d'appliquer les principes établis par les organisations internationales qui visent l'intégration des préoccupàtions de l'environnement dans les stratégies de développement durable. Dans le cadre de la Conférence de Rio, les principes suivants ont été énoncés concernant le transfert de technologies Nord-Sud :

- le transfert comprend un appui au renforcement des capacités scientifiques, technologiques, professionnelles et institutionnelles dont les pays en développement ont besoin pour faire des choix éclairés par des évaluations ;

- il consiste à appliquer et gérer judicieusement les technologies, les améliorer ou les adapter à leurs propres besoins et priorités ;

7 Le transfert de technologies est le « déplacement » des équipements, des connaissances et du savoir-faire techniques sous forme matérielle (équipements) et immatérielle (brevets). 
- il doit intégrer les techniques et connaissances locales et utiliser le matériel existant localement ;

- le transfert de technologies doit être respectueux de l'environnement, mais aussi de l'intérêt des femmes et des enfants ;

- l'utilisation d'écotechnologies permet d'accroître la durabilité des ressources ; - enfin, le transfert doit promouvoir la préservation des espèces.

La politique suisse, définie par la Conseillère fédérale Ruth Dreifuss, « considère que c'est en premier lieu au secteur privé qu'il incombe d'assurer le transfert de technologies », mais qu'il « appartient par contre aux Etats de créer les conditions cadres pour favoriser ce transfert $»^{8}$. Pour la Suisse, ces conditions cadres prioritaires sont le renforcement des capacités humaines locales, le développement des structures institutionnelles d'accueil dans les pays en développement et l'établissement de partenariats à long terme entre les entreprises privées des pays industrialisés et celles des pays en développement.

En outre, les instruments de politique économique pourraient être mis au service de la promotion des technologies environnementales. Ainsi par exemple, les garanties ou les crédits mixtes ne seraient accordés qu'aux exportateurs de technologies qui observent des règles strictes en matière de sauvegarde de l'environnement y compris dans les pays en développement. Ou pour bénéficier de l'appui de la Confédération, les entreprises s'engageraient à ne transférer que des technologies qui correspondent aux normes nationales ou internationales les plus sévères. Elles appliqueraient ainsi une sorte de «clause de l'environnement le plus favorisé " (c'est-à-dire la norme la plus favorable en matière de protection de l'environnement). Certaines entreprises multinationales se conforment déjà aux normes internes qui sont alignées sur la législation la plus exigeante.

Il convient de noter cependant que de nombreux obstacles pratiques se dressent devant la diffusion des technologies de l'environnement dans les pays en développement. ${ }^{9}$ Il s'agit notamment :

- de la faiblesse ou de l'inexistence d'un cadre législatif qui fixe les normes environnementales ;

- des difficultés d'obtenir le financement nécessaire aux investissements dans ces technologies ;

- de l'absence d'incitations fiscales pour favoriser l'acquisition des technologies environnementales par rapport aux technologies polluantes (qui sont souvent meilleur marché) ;

- du coût élevé des brevets des technologies en général et des technologies environnementales en particulier ;

- du manque de participation financière du pouvoir public ;

- de l'insuffisance d'encadrement institutionnel et de capacités humaines.

Force est de constater que même si une législation environnementale existe dans certains pays, son application se heurte souvent au manque de contrôle, au laxisme ou, plus prosaïquement, à la corruption. Un témoin, revenant de la Chine, atteste que la pollution industrielle a atteint des proportions inquiétantes

${ }^{8}$ Déclaration de la Conseillère fédérale Ruth Dreifuss lors de la Troisième séance de la Commission du développement durable, Réunion de Haut Niveau, New York, 26-28 avril 1995.

9 Cf. Programme on Technology and Environment, Trade Issues in the Transfert of Clean Technologies. OCDE, Paris, 1992. (OCDE/GD (92) 93) et OCDE, Pour un transfert de technologie, une coopération et une création de capacités efficaces en faveur d'un développement durable : document de synthèse commun. Documents de travail de l'OCDE, $\mathrm{n}^{\circ}$ 75, Paris, 1994. 
dans les zones industrielles de Pékin et de Shanghai, mais les autorités n'interviennent pas parce qu'elles ne veulent pas décourager les investissements industriels étrangers en imposant des règles antipollution. ${ }^{10}$

La concurrence internationale constitue aussi une entrave à la diffusion des technologies environnementales. Les investisseurs renoncent souvent à offrir des technologies respectueuses de l'environnement aux pays en développement au moment où elles sont plus chères que les technologies traditionnelles. Par exemple, pour obtenir une commande de construction de centrale thermique en Chine, $\mathrm{ABB}$ a renoncé à une technologie moins polluante, mais plus chère, que la technologie traditionnelle. Afin de promouvoir la diffusion de la technologie de l'environnement, il serait judicieux de prendre en charge, dans le cadre de la coopération au développement, le financement de la différence des prix entre les deux technologies.

Il a fallu attendre la création d'un groupe de travail spécial du Comité interdépartemental pour le suivi de Rio (CI Rio) pour établir une synthèse de la politique suisse sur cette question. Ce Comité, créé en mars 1993, regroupe dix-sept offices fédéraux, dont les plus importants sont la Direction du développement et de la coopération (DDC), l'Office fédéral de l'environnement, des forêts et du paysage (OFEFP) et l'Office fédéral des affaires économiques extérieures (OFAEE). Il maintient en outre le lien avec les représentants de la société civile (ONG, milieux économiques et académiques).

Son mandat est défini de la manière suivante :

- concrétiser le principe de développement durable et l'intégrer dans les politiques sectorielles de la Suisse ;

- servir de coordinateur entre différents départements et harmoniser les politiques dans les domaines concernés par la mise en œuvre des résolutions de la Conférence de Rio ;

- harmoniser les propositions défendues par la Suisse dans les organisations internationales pour l'application des décisions de Rio ;

- nouer des contacts avec les milieux extérieurs concernés par le concept de développement durable en Suisse.

Le CI Rio a institué sept Groupes de travail dont le but est de fournir des idées de solutions à l'intention des décideurs administratifs et politiques (forêts, biodiversité, changements climatiques, internalisation des coûts, cohérence de la législation et des subventions, forum de commerce et environnement, et transfert de technologies).

La principale originalité du processus de suivi de Rio réside dans son caractère transversal interne qui relie les différents départements et offices fédéraux et dans son ouverture vers les milieux extérieurs. Dans les Groupes de travail sectoriels, les représentants de l'administration, des milieux économiques, scientifiques et des ONG ont travaillé ensemble pour élaborer les différents volets faisant partie d'un plan d'action de développement durable de la Suisse.

Ainsi par exemple, le Groupe de travail transfert de technologies et coopération technologique a pu présenter un document de synthèse qui a "codifié » la politique suisse en la matière et établi un catalogue des mesures pour promouvoir le

${ }^{10}$ Arm Jean-Philippe, Hong-Kong, Shanghai, Beijing : Triangle d'or ou miroir aux alouettes, Fondation pour une $6^{6}$ Suisse responsable $(\mathrm{CH}+6)$, Neuchâtel, décembre 1996. 
transfert de technologies de l'environnement dans les pays en développement ${ }^{11}$. Les propositions suivantes ont été élaborées et avancées aussi bien par le Groupe que par les différentes instances internationales :

口 Améliorer l'accès aux technologies de l'environnement que détiennent les pays du Nord, notamment :

- mettre à disposition des pays en développement les technologies environnementales non brevetées, en particulier celles qui sont développées par des pouvoirs publics (par exemple, les technologies de traitement de déchets urbains);

- faciliter l'acquisition des technologies de l'environnement brevetées par les pays en développement, en achetant les brevets dans le cadre de la coopération au développement et en les mettant à leur disposition à un prix préférentiel ;

- faire circuler les informations et stimuler l'échange d'expériences nationales et internationales ;

- développer l'utilisation des bases de données et les réseaux d'information en matière de technologies de l'environnement.

口 S'associer avec les pays en développement pour mettre en place un cadre juridique (législatif et réglementaire) et institutionnel concernant la protection de l'environnement, afin d'encourager le développement local ou le transfert des technologies environnementales.

- Renforcer les capacités scientifiques et techniques locales (capacity building) dans le développement des technologies de l'environnement par:

- l'encadrement du transfert de technologies environnementales par la formation des acquéreurs ;

- l'augmentation de la subvention à la recherche locale en vue de développer les technologies de l'environnement dans les pays du Sud et de l'Est ;

- l'introduction des méthodes d'évaluation des technologies transférées.

- Prendre en charge, dans le cadre de la coopération au développement, la différence de prix que doit payer un pays en développement pour acquérir une technologie de l'environnement au lieu d'une technologie traditionnelle (plus polluante).

- Assurer le financement du transfert ou du développement des technologies de l'environnement (par exemple, à partir des sommes libérées par la remise de la dette des pays en développement).

La formule quadripartite de consultation, de concertation et d'élaboration des politiques sectorielles est certes intéressante et stimulante, mais elle comporte aussi ses propres limites au cas où tous les partenaires ne jouent pas le jeu et où les recommandations ne sont pas répercutées vers ou reprises par les décideurs politiques. Le rapport du Groupe de travail Transfert de technologies et coopération technologique n'a jamais obtenu l'attention qu'il méritait, il reflète pourtant assez fidèlement l'échange de vue entre les partenaires de ce groupe. En fait, le document, après avoir été désavoué par l'Union suisse du commerce et de l'industrie (VORORT), s'est égaré dans les dédales des instances administratives et politiques. Pour expliquer cette inertie, relevons la méfiance viscérale qu'ont manifestée les représentants du patronat à l'égard des autres partenaires, en par-

\footnotetext{
${ }^{11}$ Cf. Inter-Departemental Committee Rio (IDC RIO), Transfer and Cooperation in the Area of Environmentally Sound Technologies. Final Report of the IDC Rio Working Group Technology Transfer/Cooperation, Bern, September 1995. (Schlussbericht der Arbeitsgruppe IDA-Rio für die Förderung des Transfer und der Kooperation im Bereich umweltfreundlicher Technologies). Cf. aussi Le réchauffement planétaire et la Suisse : bases d'une stratégie nationale. Rapport du Groupe de travail interdépartemental sur l'évolution du système climatique, Berne, janvier 1994.
} 
ticulier des ONG, leur peur de se voir imposer une politique qui ne respecte pas les intérêts de l'économie. Mais l'administration fédérale n'était elle non plus pas trop pressée de diffuser une étude qui, en définissant une politique, pouvait aller à l'encontre des vues des divers offices fédéraux et révéler leurs divergences en matière de politique de transfert de technologies de l'environnement. Depuis, le Groupe a été dissous sans que la Suisse soit parvenue à une politique cohérente en matière de transfert de technologies environnementales.

En conclusion, on peut affirmer que les grandes déclarations politiques seront sans effet et ne seront pas appliquées tant que tous les acteurs ne sont pas convaincus que la diffusion et le transfert de technologies environnementales constituent un enjeu essentiel du développement durable, autant au Nord qu'au Sud. 